\title{
DISTRIBUTION AND ANTIMICROBIAL SUSCEPTIBILITY PATTERNS OF ClOSTRIDIUM DIfFICILE PCR RIBOTYPES IN ENGLISH HOSPITALS, 2007-08
}

\author{
J S Brazier (brazier@cardiff.ac.uk)1, R Raybould¹, B Patel², G Duckworth ${ }^{3}$, A Pearson ${ }^{3}$, A Charlett ${ }^{3}$, B I Duerden ${ }^{4}$, the HPA \\ Regional Microbiology Network ${ }^{2}$ \\ 1. Anaerobe Reference Laboratory, University Hospital of Wales, Cardiff, United Kingdom \\ 2. Regional Microbiology Network, Health Protection Agency, London, United Kingdom \\ 3. Centre for Infections, Health Protection Agency, London, United Kingdom \\ 4. Department of Health, London, United Kingdom
}

A surveillance study designed to provide a representative sample of the strains of Clostridium difficile causing infections in hospitals in England was in operation from April 2007 to the end of March 2008. Six hundred and seventy-seven isolates were obtained from 186 hospitals in the nine geographical regions of England as recognised by the Health Protection Agency's Regional Microbiology Network. Typing studies revealed that PCR ribotype 027 is now the most common strain isolated from symptomatic patients, accounting for over $41.3 \%$ of isolates in English hospitals. Type 106 was the second most common strain (20.2\%) and Type 001, which was once the most common strain associated with hospital outbreaks, has now been reduced to only $7.8 \%$ of the total. A mixture of 44 other PCR ribotypes accounted for the remaining $28.9 \%$ of isolates. This represents a changing distribution of strains when compared to a previous study performed two years earlier which showed roughly equal proportions of types 106, 001 and 027. Antimicrobial susceptibility testing by the E test method revealed significantly lower susceptibility to metronidazole in the more common strains when compared to the less common ribotypes, although none were classified as clinically resistant. Similarly, no resistance to vancomycin was detected. However, common PCR ribotypes were more resistant to moxifloxacin and erythromycin than the less common strains, which may indicate a selective advantage for resistance to these agents, and combined resistance to these two agents was a good indicator of a common ribotype.

\section{Introduction}

Hospital-acquired infections due to Clostridium difficile are a major cause of morbidity and mortality in many European countries. The problem is quite acute in the United Kingdom (UK) and the UK government's Department of Health has launched a variety of programmes aimed at tackling the rising number of such in England. One such initiative is an ongoing surveillance scheme to monitor those strains that actually cause disease and to determine their antimicrobial susceptibility patterns. This scheme is run under the auspices of the Regional Microbiology Network of the Health Protection Agency (HPA) in England and the Anaerobe Reference Laboratory (ARL) of the National Public Health Service for Wales.
The first study performed in 2005 showed that three PCR ribotypes known as Types 106, 027 and 001, in roughly equal proportions, were responsible for approximately $75 \%$ of all cases of $C$. difficile infection [1]. This second study was designed to identify whether the distribution of strains was changing, or if it was stable.

Materials and methods

The nine HPA regions took part in the programme that covered the whole of England but did not include Scotland, Wales or Northern Ireland; these run their own surveillance schemes. To collect a statistically valid number of isolates, a sampling framework was drawn up to obtain $C$. difficile isolates from toxin-positive stools from acute hospitals identified within each region that had active cases of $C$. difficile infection. Each of the 52 participating hospitals was allocated one week for sampling within the 12-month study period. The hospitals sampled a maximum of ten toxin-positive stools and submitted them to a Regional HPA laboratory for culture. Sometimes hospitals detected fewer than ten or no cases in their allotted week. No patient data were required and there was no working hypothesis. Putative isolates of $C$. difficile were then referred to the ARL at the University Hospital of Wales in Cardiff for confirmation, PCR ribotyping and susceptibility testing.

The acute hospitals selected to take part in the study by the Regional HPA network tested stool samples for toxins of $C$. difficile by their own chosen methods. Toxin-positive samples were then sent to the nearest Regional HPA laboratory for $C$. difficile culture using a national HPA Standard Operating Procedure [2]. Putative isolates of $C$. difficile were submitted without patient details but with reference numbers identifying both the originating and regional laboratories in batches to the ARL in Cardiff.

Isolates were confirmed as $C$. difficile by a combination of their characteristic odour, colonial fluorescence under long wave ultraviolet light and agglutination of a latex antibody reagent to somatic antigens of $C$. difficile (Microscreen Ltd) [3]. Isolates confirmed as C. difficile were then typed by the PCR ribotyping method developed 
in Cardiff [4] and compared to the library of ribotypes held by the ARL which currently stands at around 200 types [5].

For the convenience of the methodology involved and to permit testing of many small batches of the isolates as they were received, susceptibility to eight antibiotics was determined using the $E$ test method with an inoculum of McFarland standard 5.0 on Fastidious Anaerobe Agar (Oxoid Ltd) incubated for 48 hours. The antibiotics tested were: metronidazole, vancomycin, erythromycin, imipenem, moxifloxacin, co-amoxyclavulanic acid, penicillin and piperacillintazobactam. Minimum inhibitory concentrations of each antibiotic were recorded for each isolate and the minimum inhibitory concentration $\left(\mathrm{MIC}_{50}\right.$, the antibiotic concentration to which $50 \%$ of the tested strains are susceptible, and $\mathrm{MIC}_{90}, 90 \%$ susceptible) values calculated for each combination of drug and PCR ribotype. Differences in MIC between common and less common types were assessed for statistical significance by Student's unpaired t test

\section{Results}

The figure shows the national distribution of PCR ribotypes identified amongst the 677 isolates obtained in the study. Compared to the results in 2005 there was a $15.4 \%$ increase in the percentage of cases due to Type 027, taking it to just over $41 \%$. The percentage of Type 001 cases had fallen by $17.3 \%$ to $7.8 \%$ and of Type 106 by $6 \%$ to $20.2 \%$. Forty-four less common strains accounted for $28.9 \%$ of the total, an increase of $6.7 \%$ on the figures from 2005.

\section{F I G U R E}

National distribution of PCR ribotypes in England, 2007-08 $(n=677)$

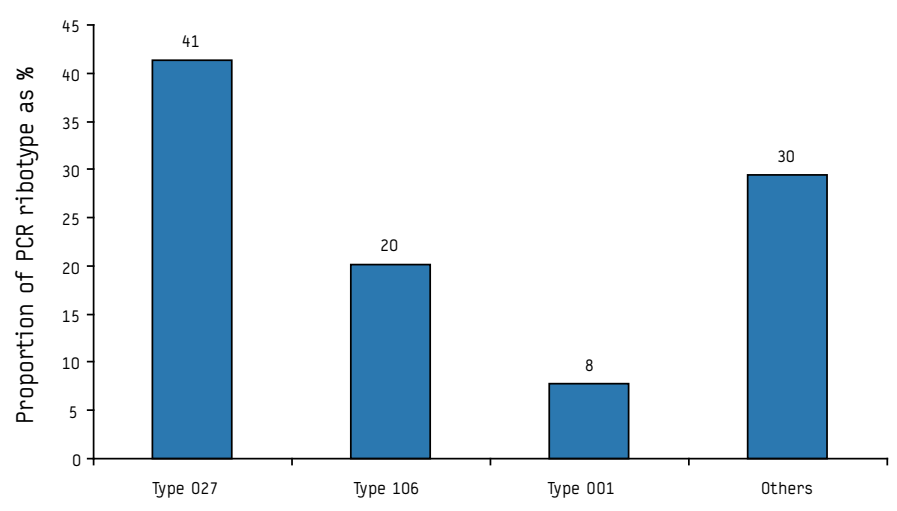

T A B L E 1

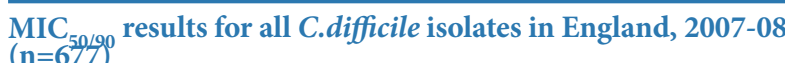

\begin{tabular}{|l|c|c|c|}
\hline & $\begin{array}{c}\text { MIC }_{50} \\
{\left[\mathrm{mg}^{\prime} \mathrm{l}\right]}\end{array}$ & $\begin{array}{c}\text { MIC }_{90} \\
{[\mathrm{mg} / \mathrm{l}]}\end{array}$ & $\begin{array}{c}\text { RANGE } \\
{[\mathrm{mg} / \mathrm{l}]}\end{array}$ \\
\hline Metronidazole $(16 \mathrm{mg} / \mathrm{l})$ & 0.38 & 1.0 & $0.064-4.0$ \\
\hline Vancomycin $(4 \mathrm{mg} / \mathrm{l})$ & 0.5 & 1.0 & $0.19-3.0$ \\
\hline Erythromycin $(4 \mathrm{mg} / \mathrm{l})$ & $>256$ & $>256$ & $0.032->256$ \\
\hline Imipenem $(16 \mathrm{mg} / \mathrm{l})$ & $>32$ & $>32$ & $0.75->32$ \\
\hline Moxifloxacin $(4 \mathrm{mg} / \mathrm{l})$ & $>32$ & $>32$ & $0.5->32$ \\
\hline Co-amoxyclav $(16 \mathrm{mg} / \mathrm{l})$ & 0.38 & 0.75 & $0.094-3.0$ \\
\hline Penicillin $(2 \mathrm{mg} / \mathrm{l})$ & 1.0 & 4.0 & $0.38->32$ \\
\hline Piperacillin-tazobactam (128mg/l) & 4.0 & 8.0 & $0.5-32.0$ \\
\hline
\end{tabular}

T A B L E 2

MICs of the five most common PCR ribotypes of $C$. difficile, England, 2007-08 ( $n=$ number of each PCR ribotype tested)

\begin{tabular}{|c|c|c|c|c|}
\hline Metronidazole & $\mathrm{MIC}_{50}[\mathrm{mg} / \mathrm{l}]$ & $\mathrm{MIC}_{90}[\mathrm{mg} / \mathrm{l}]$ & RANGE [mg/l] & $\mathrm{n}$ \\
\hline \multicolumn{5}{|l|}{ Metronidazole } \\
\hline Type 001 & 0.38 & 0.75 & $0.094-2.0$ & 53 \\
\hline Type 027 & 0.5 & 1.0 & $0.094-4.0$ & 280 \\
\hline Type 106 & 0.5 & 1.0 & $0.064-3.0$ & 137 \\
\hline Type 002 & 0.125 & 0.25 & $0.032-0.25$ & 27 \\
\hline Type 015 & 0.19 & 0.25 & $0.032-0.25$ & 25 \\
\hline Others (range only) & & & $0.047-0.5$ & 155 \\
\hline \multicolumn{5}{|l|}{ Vancomycin } \\
\hline Type 001 & 0.75 & 2.0 & $0.38-3.0$ & 53 \\
\hline Type 027 & 0.5 & 0.75 & $0.19-2.0$ & 280 \\
\hline Type 106 & 0.5 & 1.0 & $0.25-2.0$ & 137 \\
\hline Type 002 & 0.5 & 1.0 & $0.38-1.0$ & 27 \\
\hline Type 015 & 0.75 & 0.75 & $0.032-1.0$ & 25 \\
\hline Others (range only) & & & $0.19-1.0$ & 155 \\
\hline \multicolumn{5}{|l|}{ Erythromycin } \\
\hline Type 001 & $>256$ & $>256$ & $0.75->256$ & 53 \\
\hline Type 027 & $>256$ & $>256$ & $1.5->256$ & 280 \\
\hline Type 106 & $>256$ & $>256$ & $256->256$ & 137 \\
\hline Type 002 & 1.5 & 2.0 & $0.5-3.0$ & 27 \\
\hline Type 015 & 2.0 & 2.0 & $0.19->256$ & 25 \\
\hline Others (range only) & & & $0.75->256$ & 155 \\
\hline \multicolumn{5}{|l|}{ Imipenem } \\
\hline Type 001 & $>32$ & $>32$ & $6.0->32$ & 53 \\
\hline Type 027 & $>32$ & $>32$ & $2.0->32$ & 280 \\
\hline Type 106 & $>32$ & $>32$ & $2.0->32$ & 137 \\
\hline Type 002 & $>32$ & $>32$ & $4.0->32$ & 27 \\
\hline Type 015 & $>32$ & $>32$ & $2.0->32$ & 25 \\
\hline Others (range only) & & & $0.75->32$ & 155 \\
\hline \multicolumn{5}{|l|}{ Moxifloxacin } \\
\hline Type 001 & $>32$ & $>32$ & $0.75->32$ & 53 \\
\hline Type 027 & $>32$ & $>32$ & $1.0->32$ & 280 \\
\hline Type 106 & $>32$ & $>32$ & $4.0->32$ & 53 \\
\hline Type 002 & 1.0 & 2.0 & $0.75-3.0$ & 27 \\
\hline Type 015 & 1.0 & 2.0 & $0.5-12.0$ & 25 \\
\hline Others (range only) & & & $0.75->32$ & 155 \\
\hline \multicolumn{5}{|l|}{ Co-amoxyclav } \\
\hline Type 001 & 0.25 & 0.38 & $0.125-0.5$ & 53 \\
\hline Type 027 & 0.5 & 0.75 & $0.19-1.5$ & 280 \\
\hline Type 106 & 0.38 & 0.75 & $0.19-3.0$ & 137 \\
\hline Type 002 & 0.25 & 0.5 & $0.125-0.75$ & 27 \\
\hline Type 015 & 0.38 & 0.5 & $0.19-0.75$ & 25 \\
\hline Others (range only) & & & $0.094-1.0$ & 155 \\
\hline \multicolumn{5}{|l|}{ Penicillin } \\
\hline Type 001 & 1.0 & 1.5 & $0.5-3.0$ & 53 \\
\hline Type 027 & 2.0 & 4.0 & $0.5->32$ & 280 \\
\hline Type 106 & 0.75 & 4.0 & $0.38->32$ & 137 \\
\hline Type 002 & 0.75 & 1.0 & $0.38-1.5$ & 27 \\
\hline Type 015 & 1.0 & 1.5 & $0.5-3.0$ & 25 \\
\hline Others (range only) & & & $0.38-1.0$ & 155 \\
\hline \multicolumn{5}{|c|}{ Piperacillin-Tazobactam } \\
\hline Type 001 & 3.0 & 4.0 & $0.5-8.0$ & 53 \\
\hline Type 027 & 6.0 & 8.0 & $1.0-24.0$ & 280 \\
\hline Type 106 & 4.0 & 8.0 & $1.0-32.0$ & 137 \\
\hline Type 002 & 4.0 & 6.0 & $1.5-8.0$ & 27 \\
\hline Type 015 & 4.0 & 6.0 & $1.5-12.0$ & 25 \\
\hline Others (range only) & & & $0.75-12.0$ & 155 \\
\hline
\end{tabular}


Table 1 lists the range of MICs and the MIC50/90 values obtained for the eight antimicrobials tested and (in brackets) the susceptibility breakpoints chosen for each antibiotic. There was no clinical resistance to the drugs of choice for treatment (metronidazole and vancomycin) but high levels of resistance to macrolide, fluoroquinolone and carbapenem agents.

Table 2 lists the MIC values of the five most common PCR ribotypes for each of the eight antibiotics. A control strain of $\mathrm{C}$. perfringens (NCTC 11209) was used to control each drug in each batch of $E$ tests, and the MIC results for this organism never varied by more than one dilution for any of the drugs.

High levels of resistance to erythromycin and moxifloxacin were noted among the common $C$. difficile types $(027,106,001)$. Imipenem shows poor activity against all types, whilst co-amoxyclav is highly active against all types.

When analysing the MIC results for metronidazole it was noticed that the MIC values for the three most common $C$. difficile strains, namely Types 027, 106 and 001, appeared higher than those for other PCR ribotypes. The median and mean MIC values of metronidazole were calculated for each of the top ten most common strains and are listed in Table 3.

The difference in mean MICs of metronidazole for the most common PCR ribotypes 027, 106 and 001, compared to types 002, 005, 014, 015, 020, 023, 078, was $0.410 \mathrm{mg} / \mathrm{l}$. This difference between common and uncommon types was statistically significant $(p<0.0001)(95 \%$ confidence interval $(\mathrm{Cl}) 0.333-0.488)$ in the unpaired t test statistical analysis.

\section{Discussion}

Compared to a previous study analysing 881 isolates from England obtained in a similar manner in 2005 to 2006 [1], the same three strains of $C$. difficile are predominant but their proportions have changed. The most noticeable change was a drop of over $17 \%$ in the prevalence of Type 001, which decreased from $25.1 \%$ to $7.8 \%$. The incidence of Type 027 rose from $25.9 \%$ to $41.3 \%$, an increase of $15.4 \%$ and Type 106 decreased by $6 \%$ from $26.2 \%$ to $20.2 \%$. The incidence of other PCR ribotypes rose to $29.5 \%$ These results are broadly in agreement with a previous unstructured sampling of ribotypes in English hospitals [6].

T A B L E 3

Median and mean MIC values of PCR ribotypes to metronidazole (Mz), England, 2007-08

\begin{tabular}{|l|c|c|}
\hline & Mean Mz MIC [mg/l] & Median Mz MIC [mg/l] \\
\hline Type 001 $(n=53)$ & 0.5 & 0.38 \\
\hline Type 027 $(n=280)$ & 0.61 & 0.5 \\
\hline Type 106 $(n=137)$ & 0.58 & 0.5 \\
\hline Type 002 $(n=27)$ & 0.14 & 0.125 \\
\hline Type 005 $(n=14)$ & 0.16 & 0.19 \\
\hline Type 014 $(n=20)$ & 0.18 & 0.19 \\
\hline Type 015 $(n=25)$ & 0.18 & 0.19 \\
\hline Type 020 $(n=17)$ & 0.20 & 0.19 \\
\hline Type 023 $(n=13)$ & 0.09 & 0.094 \\
\hline Type 078 $(n=15)$ & 0.13 & 0.125 \\
\hline
\end{tabular}

The emergence and spread of Type 027 in England may be an indication of what may happen in other countries where this strain has been detected since it was first reported in North America and soon after emerged in Stoke Mandeville Hospital in England in 2004. Eurosurveillance has published a number of articles tracking its incidence in outbreaks across Europe [6-8], but to date nationwide surveillance has been conducted only in England to reveal the accurate distribution of this and other ribotypes across the nation. Looking at reports from other European countries [6] it is of interest to note that Type 106 is virtually unique to the UK, although the reason for this is unknown. In England, some regional variation in the distribution of strains has been noticed. For example, Type 001 was the most common isolate in the North East Region of England, but was not found in the East Midlands Region, whereas theYorkshire and Humberside Region showed a greater variety of different ribotypes than any other region.

The breakpoints listed for erythromycin and moxifloxacin (see antibiogram in Table 2) showed widespread resistance amongst the common ribotypes. Importantly, the MIC levels for the antibiotics of choice for treatment (metronidazole and vancomycin) were not indicative of clinical resistance. However, the $\mathrm{MIC}_{50}$ and $\mathrm{MIC}_{90}$ levels for metronidazole for the common PCR ribotypes 027, 106 and 001 were several dilutions higher and their MIC ranges much larger than those for the less common strains.

The mean and median MIC values to metronidazole for the ten most common PCR ribotypes listed in Table 3. suggest that metronidazole MICs are increasing in common $C$. difficile PCR ribotypes, and this should be closely monitored by further surveillance studies. A recent report by Kuijper et al. on decreased effectiveness of metronidazole treatment [10] is another warning to this effect. Baines et al. suggested that Type 001 in particular had higher MICs than the other common strains, although a different testing methodology was used [11].

There was no evidence of similar elevated MICs for vancomycin among common or non-epidemic ribotypes. Vancomycin MICs for all types ranged from 0.19 to $3.0 \mathrm{mg} / \mathrm{l}$. Common PCR ribotypes exhibited much higher MICs to moxifloxacin and erythromycin than the less common strains, which may indicate a selective advantage for resistance to fluoroquinolone and macrolide agents. Combined resistance to these agents is a good indicator of a common ribotype. Imipenem has little activity across all ribotypes, both common and uncommon, and it is probably of little value to continue testing this agent since resistance is so widespread. Co-amoxyclav had a high degree of activity against all types, with MICs ranging from 0.094 to $3.0 \mathrm{mg} / \mathrm{l}$. MICs for penicillin ranged from 0.38 to over $32 \mathrm{mg} / \mathrm{l}$, but resistance to penicillin did not appear to be related to type. Piperacillin-tazobactam MICs ranged from 0.5 to $32 \mathrm{mg} / \mathrm{L}$ and the highest values were seen in Type 106.

A limitation of this study is the omission of clindamycin susceptibility data that would have been of interest to compare the susceptibility of Type 027 isolates in the UK with data from other countries. This agent was excluded because it is rarely used in the UK. Nor was it possible to determine seasonal variations since each hospital was allocated only one week to collect toxin-positive stools during the 12-month study period.

The above data fulfil the primary objectives of the study, which were to establish the distribution of the types of $C$. difficile 
causing infections in English hospitals and to obtain data on their antimicrobial susceptibilities. These data are of value in our understanding of which strains are dominant in English hospitals, which antimicrobial agents are important in terms of treatment, and which of them may be important in applying antibiotic selective pressure.

A third one-year study funded by the UK Department of Health has just begun testing the same set of antibiotics, and it will be of interest to see if the distribution pattern of PCR ribotypes will change yet again.

\section{Acknowledgements}

We are grateful for the technical assistance of Carol Davis of the Anaerobe Reference Laboratory and to all the diagnostic microbiology departments for providing and testing the stool samples and to staff of the regional HPA laboratories without whom the data could not have been collected.

\section{References}

1. Brazier JS, Patel B, Pearson A. Distribution of Clostridium difficile PCR ribotype 027 in British hospitals. Euro Surveill. 2007;12(17):pii=3182. Available from: http://www.eurosurveillance.org/ViewArticle.aspx?ArticleId=3182.

2. Health Protection Agency. National Standard Methods. Available from: www. hpa-standardmethods.org.uk/pdf_sops.asp

3. Brazier JS. The diagnosis of Clostridium difficile-associated disease. J Antimicrob Chemother. 1998;41 Suppl C:29-40.

4. O'Neill GL, Ogunsola FT, Brazier JS, Duerden BI. Modification of a PCR ribotyping method for application as a routine typing scheme for Clostridium difficile. Anaerobe, 1996;2(4):205-9.

5. Stubbs SLJ, Brazier JS, O'Neill GL, Duerden BI. PCR targeted to the 16S-23S rRNA gene intergenic spacer region of Clostridium difficile and construction of a library consisting of 116 different PCR ribotypes. J Clin Microbiol. 1999;37(2):461-3.

6. Kuijper EJ, Barbut F, Brazier JS, Kleinkauf N, Eckmanns T, Lambert ML, et al. Update of Clostridium difficile infection due to PCR ribotype 027 in Europe, 2008. Euro Surveill. 2008;13(31):pii=18942. Available from: http://www. eurosurveillance.org/ViewArticle.aspx?ArticleId $=18942$

7. Kleinkauf N, Weiss B, Jansen A, Eckmanns T, Bornhofen B, Kuehnen E, et al. Confirmed cases and report of clusters of severe infections due to Clostridium difficile PCR ribotype 027 in Germany. Euro Surveill. 2007;12(46):pii=3307. Available from: http://www.eurosurveillance.org/ ViewArticle.aspx?ArticleId $=3307$

8. Lyytikäinen $\mathrm{O}$, Mentula S, Kononen E, Kotila S, Tarkka E, Anttila VJ, et al. First isolation of Clostridium difficile PCR ribotype 027 in Finland. Euro Surveill. 2007;12(45):pii=3303. Available from: http://www.eurosurveillance. org/ViewArticle.aspx?ArticleId $=3303$

9. Long S, Fenelon L, Fitzgerald S, Nolan N, Burns K, Hannan M, et al. First isolation and report of clusters of Clostridium difficile PCR 027 cases in Ireland. Euro Surveill. 2007;12(17):pii=3183. Available from: http://www. eurosurveillance.org/ViewArticle.aspx?ArticleId=3183

10. Kuijper EJ. Wilcox MH. Decreased effectiveness of metronidazole treatment of Clostridium difficile infection? : Clin Infect Dis. 2008;47(1):63-5.

11. Baines SD, O'Connor R, Freeman J, Fawley WN, Harmanus C, Mastrantonio P, et al. Emergence of reduced susceptibility to metronidazole in Clostridium difficile. J Antimicrob Chemother. 2008; doi:10.1093/jac/dkn313. antimicrobial susceptibility patterns of Clostridium difficile PCR ribotypes in English hospitals, 2007-08. Euro Surveill. 2008;13(41):pii=19000. Available online: http://www. eurosurveillance.org/ViewArticle.aspx?ArticleId $=19000$ 
